\title{
Large-Scale Multipair Two-Way Relay Networks with Distributed AF Beamforming
}

\author{
Hien Quoc Ngo and Erik G. Larsson
}

\section{Linköping University Post Print}

\section{Tweet}

N.B.: When citing this work, cite the original article.

(C2013 IEEE. Personal use of this material is permitted. However, permission to reprint/republish this material for advertising or promotional purposes or for creating new collective works for resale or redistribution to servers or lists, or to reuse any copyrighted component of this work in other works must be obtained from the IEEE.

Hien Quoc Ngo and Erik G. Larsson, Large-Scale Multipair Two-Way Relay Networks with Distributed AF Beamforming, 2013, IEEE Communications Letters.

Postprint available at: Linköping University Electronic Press http://urn.kb.se/resolve?urn=urn:nbn:se:liu:diva-98122 


\title{
Large-Scale Multipair Two-Way Relay Networks with Distributed AF Beamforming
}

\author{
Hien Quoc Ngo, Student Member, IEEE, and Erik G. Larsson, Senior Member, IEEE
}

\begin{abstract}
We consider a multipair two-way relay network where multiple communication pairs simultaneously exchange information with the help of multiple relay nodes. All nodes are equipped with a single antenna and channel state information is available at the relay nodes. Each relay uses very simple signal processing in a distributed manner, called distributed amplifyand-forward $(A F)$ relaying. A closed-form expression for the achievable rate is derived. We show that the distributed AF scheme outperforms conventional orthogonal relaying. When the number of relays is large, the distributed AF relaying scheme can achieve the capacity scaling given by the cut-set upper bound. Furthermore, when the number of relays grows large, the transmit powers of each terminal and of the relay can be made inversely proportional to the number of relays while maintaining a given quality-of-service. If the transmit power of each terminal is kept fixed, the transmit power of each relay can be scaled down inversely proportional to the square of the number of relays.
\end{abstract}

\section{INTRODUCTION}

The multipair one-way relay channel, where multiple sources simultaneously transmit signals to their destinations through the use of a multiple relay nodes, has attracted substantial interest [1]-[3]. In [1], [2], the authors proposed a transmission scheme where the beamforming weights at the relays are obtained under the assumption that all relay nodes can cooperate. A simple distributed beamforming scheme that requires only local channel state information (CSI) at the relays, and which performs well with a large number of relay nodes, was proposed in [3]. With one-way protocols, the halfduplex (HD) constraint at the relays imposes a pre-log factor $1 / 2$ for the data rate and hence, limits the spectral efficiency. To overcome this spectral efficiency loss in the one-way relay channel, the multipair two-way relay channel has recently been considered [4], [5]. However, those studies considered multipair systems where only one relay (equipped with multiple antennas) participates in the transmission. Multiple singleantenna relays supporting multiple communication pairs were considered in [6]-[8]. In [6], the weighting coefficient at each relay was designed to minimize the transmit power at the relays under a given received signal-to-interference-plus-noise ratio constraint at each terminal. By contrast, the objective function of [7] was the the sum rate. These works assume that there is a central processing center. A distributed beamforming scheme where the relay weighting coefficient is designed at

The associate editor coordinating the review of this paper and approving it for publication was Dr. M. Elkashlan.

The authors are with the Department of Electrical Engineering (ISY), Linköping University, 58183 Linköping, Sweden (email: nqhien@isy.liu.se; egl@isy.liu.se).

This work was supported in part by the Swedish Research Council (VR), the Swedish Foundation for Strategic Research (SSF), and ELLIIT. each relay was proposed in [8]. However, [8] assumed that each relay has CSI of all relay-terminal pairs. This requires cooperation between the relay nodes for the CSI exchange.

In this paper, we propose and analyze a distributed amplifyand-forward (AF) relaying scheme for multipair two-way relay channels which does not require cooperation between the relay nodes. Our scheme is suitable for dense networks where there are many idle nodes willing to act as relays. Here, we assume that the relays have perfect knowledge of local CSI, that is of the channels from each terminal to the relay. The fundamental basis of our proposed scheme is that when the number of relays is large, the channel vectors between the terminals and relays are pairwisely nearly orthogonal. There is also empirical support for the near-orthogonality assumption, most notably in the large scale MIMO literature [9]. This makes it possible for the relays to use very simple signal processing.

The work that is most closely related to this paper is [3]. In [3] the authors investigated the scaling law of the power efficiency in the multipair one-way relay channel. By contrast, here, we consider the two-way relay channel. We derive a closed-form expression of the achievable rate. The resulting expression is simple and yields useful insight. We show that when the number of relays $M \rightarrow \infty$, the distributed $\mathrm{AF}$ relaying achieves the cut-set upper bound on the capacity. Furthermore, when $M$ is large, we achieve the following power scaling laws: (i) the transmit powers of each terminal and of each relay can be scaled $\propto 1 / M$ with no performance reduction; and (ii) if the transmit power of each terminal is fixed, the transmit power of each relay can be scaled $\propto 1 / M^{2}$.

\section{Multipair Two-Way Relay Channel Model}

Consider a network in which $K$ communication pairs $\left(\mathrm{T}_{1, k}, \mathrm{~T}_{2, k}\right), k=1, \ldots, K$, share the same time-frequency resource. Two terminals $\mathrm{T}_{1, k}$ and $\mathrm{T}_{2, k}$ exchange their information with the help of $M$ relay nodes $\mathrm{R}_{m}, m=1,2, \ldots, M$. Typically, $K \ll M$. All nodes are equipped with a single antenna and use HD operation. We assume that there is no direct link between $\mathrm{T}_{1, k}$ and $\mathrm{T}_{2, k}$ that can be exploited. Transmission will take place in both directions (from the terminals to the relays and back) on the same frequency, and we assume that the channels are reciprocal [9].

We further assume that the relay nodes have full CSI, while the terminals have statistical but no instantaneous CSI. The CSI at the relay nodes could be obtained by using training sequences transmitted from the terminals, at a cost of $2 K$ symbols per coherence interval. The assumption that the terminals do not have instantaneous CSI is reasonable for 


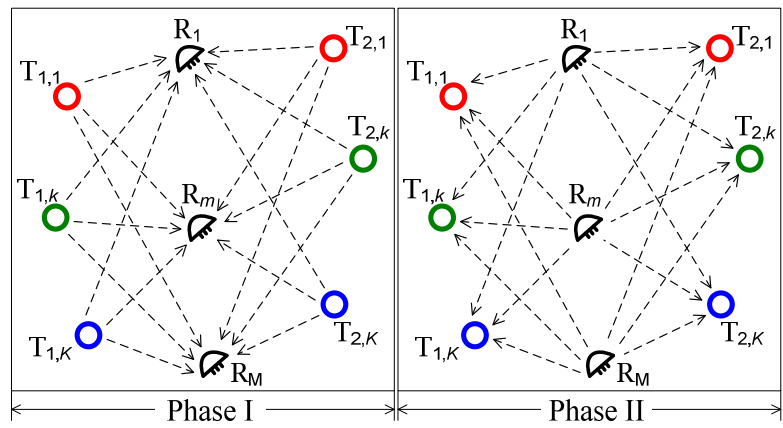

Fig. 1. Multipair Two-Way Relaying Network.

practical systems where the number of relay nodes is large. To obtain instantaneous CSI at the terminals, we would have to spend at least $M$ symbols per coherence interval. We show below that due to hardening effects, although the terminals do not have instantaneous CSI, they can near-coherently detect the signals aided by the statistical distribution of the channels.

\section{Distributed AF Transmission Scheme}

The communication occurs in two phases, as detailed next and in Fig. 1. We assume perfect time synchronization. In [3], the authors have shown that the lack of synchronicity does not have much effect on the system performance.

\section{A. Phase I}

All terminals simultaneously broadcast their signals to all relay nodes. Let $h_{m, k}$ and $g_{m, k}$ be the channel coefficients from $\mathrm{T}_{1, k}$ to $\mathrm{R}_{m}$ and from $\mathrm{R}_{m}$ to $\mathrm{T}_{2, k}$, respectively. The channel model includes small-scale fading (Rayleigh fading) and large-scale fading, i.e., $h_{m, k}=\sqrt{\alpha_{m, k}} \tilde{h}_{m, k}$ and $g_{m, k}=$ $\sqrt{\beta_{m, k}} \tilde{g}_{m, k}$, where $\tilde{h}_{m, k} \sim \mathcal{C N}(0,1), \tilde{g}_{m, k} \sim \mathcal{C N}(0,1)$. Here, $\alpha_{m, k}$ and $\beta_{m, k}$ represent the large-scale fading. Then, the received signal at $R_{m}$ is given by

$$
r_{m}=\sqrt{p_{\mathrm{S}}} \mathbf{h}_{m}^{T} \mathbf{x}_{1}+\sqrt{p_{\mathrm{S}}} \mathbf{g}_{m}^{T} \mathbf{x}_{2}+w_{m}
$$

where $\mathbf{x}_{i} \triangleq\left[\begin{array}{lll}x_{i, 1} & \ldots & x_{i, K}\end{array}\right]^{T}, \sqrt{p_{\mathrm{S}}} x_{i, k}$ is the transmitted signal from $\mathrm{T}_{i, k}$ (the average transmit power of each terminal is $p_{\mathrm{S}}$ ), $\mathbf{h}_{m} \triangleq\left[\begin{array}{lll}h_{m, 1} & \ldots & h_{m, K}\end{array}\right]^{T}, \mathbf{g}_{m} \triangleq\left[\begin{array}{llll}g_{m, 1} & \ldots & g_{m, K}\end{array}\right]^{T}$, and $w_{m}$ is AWGN at $R_{m}$. We assume that $w_{m} \sim \mathcal{C N}(0,1)$.

\section{B. Phase II - Distributed AF Relaying}

All relays broadcast scaled and phase-rotated versions of their received signals to all terminals. The basic idea of distributed AF relaying is as follows. Consider the two-way relay channel with $K$ pairs as a one-way relay channel with $2 K$ pairs where the groups of sources and destinations are the same. Then, we apply the relaying scheme for one-way relay channels in $\left[3\right.$, Sec. V]. ${ }^{1}$ We propose to let $\mathrm{R}_{m}$ transmit the following phase-rotated version of the received signal:

$$
x_{\mathrm{R}_{m}}=\gamma_{m} \mathbf{a}_{m}^{H} \mathbf{D} \mathbf{a}_{m}^{*} r_{m}
$$

where $\mathbf{a}_{m} \triangleq\left[\begin{array}{ll}\mathbf{h}_{m}^{T} & \mathbf{g}_{m}^{T}\end{array}\right]^{T}, \mathbf{D} \triangleq\left[\begin{array}{cc}\mathbf{0} & \mathbf{I}_{K} \\ \mathbf{I}_{K} & \mathbf{0}\end{array}\right]$ is used to permute the signal position to ensure that the signal transmitted

\footnotetext{
${ }^{1}$ Considering a multipair one-way relay channel with $K$ sources $\mathrm{T}_{1, k}, K$ destinations $\mathrm{T}_{2, k}, k=1, \ldots, K$, and $M$ relay nodes $\mathrm{R}_{m}, m=1, \ldots, M$, the scaled version at $R_{m}$ proposed in [3] is $\mathbf{g}_{m}^{H} \mathbf{h}_{m}^{*} r_{m}$.
}

from $\mathrm{T}_{1, k}$ arrives at its destination $\mathrm{T}_{2, k}$ and vice versa, and $\gamma_{m}$ is a normalization factor which controls the transmit power at $\mathrm{R}_{m}$, chosen such that $\mathbb{E}\left\{\left|x_{\mathrm{R}_{m}}\right|^{2}\right\}=p_{\mathrm{R}}$. Hence, ${ }^{2}$

$$
\begin{aligned}
\gamma_{m} & =\sqrt{\frac{p_{\mathrm{R}}}{\mathbb{E}\left\{\left|\mathbf{a}_{m}^{H} \mathbf{D} \mathbf{a}_{m}^{*}\right|^{2}\left(p_{\mathrm{S}}\left\|\mathbf{h}_{m}\right\|^{2}+p_{\mathrm{S}}\left\|\mathbf{g}_{m}\right\|^{2}+1\right)\right\}}} \\
& =\sqrt{\frac{p_{\mathrm{R}} / 4}{p_{\mathrm{S}} \sum_{j=1}^{K}\left(\alpha_{m, j}+\beta_{m, j}\right)\left(\alpha_{m, j} \beta_{m, j}+c_{m}\right)+c_{m}}}
\end{aligned}
$$

where $c_{m} \triangleq \sum_{i=1}^{K} \alpha_{m, i} \beta_{m, i}$, see Appendix A. Let $n_{2, k}$ be the $\mathcal{C N}(0,1)$ noise at $\mathrm{T}_{2, k}$. Then, the received signal at $\mathrm{T}_{2, k}$ is

$$
y_{2, k}=\sum_{m=1}^{M} g_{m, k} x_{\mathrm{R}_{m}}+n_{2, k}
$$

When the number of relay nodes $M$ is large, the received signal at $\mathrm{T}_{2, k}$ is dominated by the desired signal part (which includes $x_{1, k}$ ). As a result, we can obtain noise-free and interference-free communication links when $M$ grows without bound. A more detailed analysis is given in the next section.

\section{Asymptotic $(M \rightarrow \infty, K<\infty)$ Performance}

In this section, we provide basic insights into the performance of our proposed scheme when $M \rightarrow \infty$ for fixed $K$ and $p_{\mathrm{R}}$. We will show that our proposed scheme performs well when $M$ is large. From (1), (2), and (5), we have

$$
\begin{aligned}
y_{2, k} & =\underbrace{\sqrt{p_{\mathrm{S}}} \sum_{m=1}^{M} p_{m, k} x_{1, k}}_{\mathcal{L}_{1}}+\underbrace{\sqrt{p_{\mathrm{S}} \sum_{j \neq k}^{K} \sum_{m=1}^{M} p_{m, j} x_{1, j}+\sqrt{p_{\mathrm{S}} \sum_{j=1}^{K} \sum_{m=1}^{M} q_{m, j} x_{2, j}}}}_{\mathcal{L}_{2}} \\
+ & \underbrace{\sum_{m=1}^{M} \gamma_{m} g_{m, k} \mathbf{a}_{m}^{H} \mathbf{D} \mathbf{a}_{m}^{*} w_{m}+n_{2, k}}_{\mathcal{L}_{3}}
\end{aligned}
$$

where $p_{m, j} \triangleq \gamma_{m} g_{m, k} \mathbf{a}_{m}^{H} \mathbf{D} \mathbf{a}_{m}^{*} h_{m, j}$ and $q_{m, j} \triangleq$ $\gamma_{m} g_{m, k} \mathbf{a}_{m}^{H} \mathbf{D a}_{m}^{*} g_{m, j}$. Here $\mathcal{L}_{1}, \mathcal{L}_{2}$, and $\mathcal{L}_{3}$ represent the desired signal, multi-terminal interference, and noise effects, respectively. We have

$$
\mathbb{E}\left\{p_{m, k}\right\}=2 \gamma_{m} \mathbb{E}\left\{g_{m, k}\left(\sum_{i=1}^{K} g_{m, i}^{*} h_{m, i}^{*}\right) h_{m, k}\right\}=2 \gamma_{m} \alpha_{m, k} \beta_{m, k} .
$$

We assume that $\operatorname{Var}\left\{p_{m, k}\right\}, m=1, \ldots, M$, are uniformly bounded, i.e., $\exists c<\infty$ : $\operatorname{Var}\left\{p_{m, k}\right\} \leq c, \forall m, k$ [10]. Since $p_{m, k}, m=1,2, \ldots, M$, are independent, it follows from Tchebyshev's theorem [10] that

$$
\frac{1}{M} \mathcal{L}_{1}-\frac{1}{M} \sqrt{p_{\mathrm{S}}} \sum_{m=1}^{M} 2 \gamma_{m} \alpha_{m, k} \beta_{m, k} x_{1, k} \underset{M \rightarrow \infty}{\stackrel{P}{\rightarrow}} 0 .
$$

where $\stackrel{P}{\rightarrow}$ denotes convergence in probability. Similarly, since $\mathbb{E}\left\{q_{m, j}\right\}=0$ and $\mathbb{E}\left\{\gamma_{m} g_{m, k} \mathbf{a}_{m}^{H} \mathbf{D} \mathbf{a}_{m}^{*} w_{m}\right\}=0$, we have

$$
\frac{1}{M} \mathcal{L}_{2} \underset{M \rightarrow \infty}{\stackrel{P}{\rightarrow}} 0, \frac{1}{M} \mathcal{L}_{3} \underset{M \rightarrow \infty}{\stackrel{P}{\rightarrow}} 0 .
$$

We can see from (8) and (9) that, when $M$ is large, the power of the desired signal grows as $M^{2}$, while the power of the

\footnotetext{
${ }^{2}$ Note that $\gamma_{m}$ could alternatively be chosen depending on the instantaneous CSI which corresponds to having a short-term power constraint. However, we use (3) since: i) it yields a tractable form of the achievable rate which enables us to further analyze the system performance; and ii) the law of large numbers guarantees that the denominator of (3) is nearly deterministic unless $K$ is small. Thus, our choice does not substantially affect the obtained insights.
} 


$$
R_{2, k}=\frac{1}{2} \log _{2}\left(1+\frac{4 p_{\mathrm{S}}\left(\sum_{m=1}^{M} \gamma_{m} \alpha_{m, k} \beta_{m, k}\right)^{2}}{p_{\mathrm{R}} \sum_{m=1}^{M} \beta_{m, k}+4 p_{\mathrm{S}} \sum_{m=1}^{M} \sum_{j=1}^{K} \gamma_{m}^{2} \alpha_{m, k} \beta_{m, k}^{2}\left(\alpha_{m, j}+\beta_{m, j}\right)+4 p_{\mathrm{S}} \sum_{m=1}^{M} \gamma_{m}^{2} \beta_{m, k}^{2}\left(2 \alpha_{m, k} \beta_{m, k}+c_{m}+\frac{\alpha_{m, k}}{p_{\mathrm{S}}}\right)+1}\right)
$$

interference and noise grows more slowly. As a result, with an unlimited number of relay nodes, the effects of interference, noise, and fast fading disappear. More precisely, as $M \rightarrow \infty$,

$$
\frac{y_{2, k}}{M}-\sqrt{p_{\mathrm{S}}} \frac{\sum_{m=1}^{M} 2 \gamma_{m} \alpha_{m, k} \beta_{m, k}}{M} x_{1, k} \stackrel{P}{\rightarrow} 0 .
$$

The received signal includes only the desired signal and hence, the capacity increases without bound.

\section{Achievable Rate for Finite $M$}

In this section, we derive a closed-form expression of the achievable rate for finite $M$ which can be used to draw more precise quantitative conclusions about the performance of the distributed AF relaying scheme. The terminals do not have instantaneous CSI, but they know the statistical distribution of the channel. Hence, the terminals use the mean of the effective channel gain to coherently detect the desired signals [3].

Consider the link $\mathrm{T}_{1, k} \rightarrow$ Relays $\rightarrow \mathrm{T}_{2, k}$. From (6), the received signal at $\mathrm{T}_{2, k}$ can be rewritten as the desired signal $\sqrt{p_{\mathrm{S}}} \mathbb{E}\left\{\sum_{m=1}^{M} p_{m, k}\right\} x_{1, k}$ plus a remaining term which is considered as the effective noise. This effective noise is uncorrelated with the desired signal. Then, Gaussian noise is the worst case, and we obtain the following achievable rate:

$$
R_{2, k}=\frac{1}{2} \log _{2}\left(1+\frac{p_{\mathrm{S}}\left|\mathbb{E}\left\{\sum_{m=1}^{M} p_{m, k}\right\}\right|^{2}}{p_{\mathrm{S}} \operatorname{Var}\left\{\sum_{m=1}^{M} p_{m, k}\right\}+\mathrm{MT}_{k}+\mathrm{AN}_{k}}\right)
$$

where the pre-log factor of $1 / 2$ is due to the half-duplex relaying, $\operatorname{Var}\{x\}$ denotes the variance of a RV $x$, and

$$
\begin{aligned}
& \mathrm{MT}_{k}=p_{\mathrm{S}} \sum_{j \neq k}^{K} \mathbb{E}\left\{\left|\sum_{m=1}^{M} p_{m, j}\right|^{2}\right\}+p_{\mathrm{S}} \sum_{j=1}^{K} \mathbb{E}\left\{\left|\sum_{m=1}^{M} q_{m, j}\right|^{2}\right\} \\
& \mathrm{AN}_{k}=\sum_{m=1}^{M} \gamma_{m}^{2} \mathbb{E}\left\{\left|g_{m, k} \mathbf{a}_{m}^{H} \mathbf{D} \mathbf{a}_{m}^{*}\right|^{2}\right\}+1 .
\end{aligned}
$$

We now derive a closed-form expression of the achievable rate.

Proposition 1: With distributed AF relaying, the achievable rate of the communication link $\mathrm{T}_{1, k} \rightarrow$ Relays $\rightarrow \mathrm{T}_{2, k}$ is given by (14), shown at the top of the page.

Proof: See Appendix B.

\section{A. Discussion of Results}

For simplicity, we next consider a simplified case where the large-scale fading is neglected, i.e., $\alpha_{m, k}=\beta_{m, k}=1$, for all $m, k$. The same insights will be straightforwardly obtained for the case when the large-scale fading is taken into account. Substituting $\alpha_{m, k}=\beta_{m, k}=1$ into (14), we get

$$
R_{2, k}=\frac{1}{2} \log _{2}\left(1+\frac{p_{\mathrm{S}} p_{\mathrm{R}} M / K}{p_{\mathrm{S}} p_{\mathrm{R}}\left(2 K+5+\frac{2}{K}\right)+\frac{p_{\mathrm{R}}}{K}(K+1)+\frac{2 p_{\mathrm{S}}}{M}(K+1)+\frac{1}{M}}\right) .
$$

We can see that, when $M$ goes to infinity, $R_{2, k} \rightarrow \infty$. This lower bound on the rate coincides with the asymptotic (but exact) rate obtained in Section III-C and hence, the achievable rate (14) is very tight at large $M$.
1) Achievability of the Network Capacity: If $p_{\mathrm{S}}$ and $E_{\mathrm{R}}=$ $M p_{\mathrm{R}}$ (total transmit power of all relays) are fixed regardless of $M$, then $R_{2, k}=\frac{1}{2} \log _{2} M+\mathcal{O}(1)$, as $M \rightarrow \infty$. This result coincides with the one which is obtained by using the cut-set upper bound on the network capacity of MIMO relay networks where all terminals are equipped with a single antenna [11]. Note that the result obtained in [11] relies on the assumption that the relay and destination nodes have instantaneous CSI. Here, we assume that only the relays have instantaneous CSI. In particular, with our proposed technique, the sum rate scales as $K \log _{2} M+\mathcal{O}(1)$ at large $M$ which is identical to the cut-set bound on the sum capacity of our considered multipair two-way relay network. ${ }^{3}$

2) Power Scaling Laws:

(i) If $p_{\mathrm{S}}=E_{\mathrm{S}} / M$ and $p_{\mathrm{R}}=E_{\mathrm{R}} / M$, where $E_{\mathrm{S}}$ and $E_{\mathrm{R}}$ are fixed regardless of $M$, then

$$
R_{2, k} \rightarrow \frac{1}{2} \log _{2}\left(1+\frac{E_{\mathrm{S}} E_{\mathrm{R}}}{E_{\mathrm{R}}(K+1)+K}\right), \text { as } M \rightarrow \infty
$$

which implies that when $M$ is large, we can cut the transmit power $p_{\mathrm{S}} \propto 1 / M$ without any performance reduction.

(ii) If $p_{\mathrm{S}}$ and $E_{\mathrm{R}}$ are fixed regardless of $M$, and $p_{\mathrm{R}}=$ $E_{\mathrm{R}} / M^{2}$, then

$$
R_{2, k} \rightarrow \frac{1}{2} \log _{2}\left(1+\frac{p_{\mathrm{S}} E_{\mathrm{R}}}{2 p_{\mathrm{S}} K(K+1)+K}\right), \text { as } M \rightarrow \infty .
$$

We can see that when $M$ is large, the transmit power of each relay node can be reduced proportionally to $1 / M^{2}$ with no performance degradation. As a result, the transmit power of each relay node can be very small.

\section{Numerical Results AND Discussion}

In this section, we examine the sum rate of our proposed scheme. For comparison, we also consider the sum rate of multipair one-way relaying proposed in [3], and the sum rate of the conventional orthogonal scheme where the transmission of each pair is assigned different time slots or frequency bands. In addition, we consider the sum rate of our scheme but with a genie receiver (instantaneous CSI) at the terminals. For this case, the achievable rate of the link $\mathrm{T}_{1, k} \rightarrow$ Relays $\rightarrow \mathrm{T}_{2, k}$ is

$$
R_{2, k}=\frac{1}{2} \mathbb{E}\left\{\log _{2}\left(1+\frac{p_{\mathrm{S}}\left|\sum_{m=1}^{M} p_{m, k}\right|^{2}}{\mathrm{MT}_{k}+\mathrm{AN}_{k}}\right)\right\} .
$$

We choose $K=5, p_{\mathrm{S}}=10 \mathrm{~dB}$, and $\alpha_{m, k}=\beta_{m, k}=1$. We assume that the total transmit powers for the two phases are the same, i.e., $2 K p_{\mathrm{S}}=M p_{\mathrm{R}}$. Furthermore, for fair comparison, the total transmit powers of all schemes are the same.

\footnotetext{
${ }^{3}$ Suppose that all terminals $\mathrm{T}_{1, k}$ can cooperate and all terminals $\mathrm{T}_{2, k}$ can also cooperate. Then we have a two-way relay network with two terminals each equipped with $K$ antennas, and $M$ single-antenna relays. From [12], the network capacity of this resulting system is $K \log _{2} M+\mathcal{O}(1)$. Clearly, this resulting system has greater capacity than the original one. Thus, an upper bound on the sum capacity of our multipair network is $K \log _{2} M+\mathcal{O}(1)$.
} 


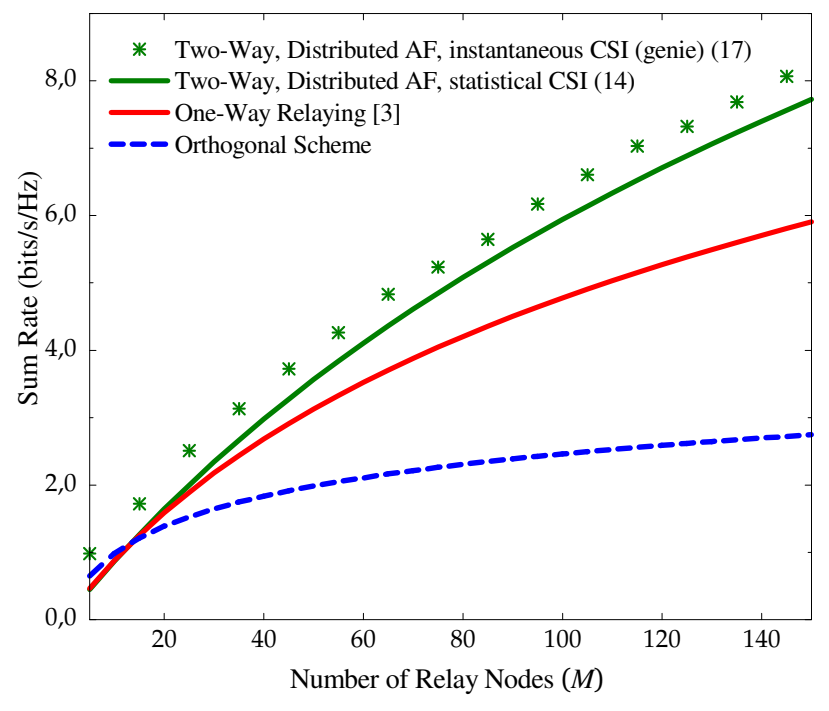

Fig. 2. Sum rate versus the number of relay nodes $\left(K=5, p_{\mathrm{S}}=10 \mathrm{~dB}\right)$.

Figure 2 shows the sum rate versus the number of relay nodes for the different transmission schemes. We can see that the number of relay nodes has a very strong impact on the performance. The sum rate increases significantly when we increase $M$. For small $M(\lesssim 10)$, owing to inter-terminal interference, our proposed scheme performs worse than the orthogonal scheme. However, when $M$ grows large, the effect of inter-terminal interference and noise dramatically reduces and hence, our proposed scheme outperforms the orthogonal scheme. Compared with the one-way relaying proposed in [3], our distributed AF relaying scheme is better, and the advantage increases when $M$ increases. The gain stems from the reduced pre-log penalty (from $1 / 2$ to 1 ), however, our scheme suffers from more interference and therefore, the gain is somewhat less than a doubling. When $M$ is small, the channels are no longer nearly orthogonal and the inter-terminal interference cannot be notably reduced and hence, our scheme is not better than the one-way relaying scheme. Furthermore, the performance gap between the cases with instantaneous (genie) and statistical CSI at the terminals is small. This implies that using the mean of the effective channel gain for signal detection is fairly good.

\section{APPENDIX}

\section{A. Derivation of (4)}

To compute $\gamma_{m}$, we need to compute $\mathbb{E}\left\{\left|\mathbf{a}_{m}^{H} \mathbf{D a} \mathbf{a}_{m}^{*}\right|^{2}\right\}$, $\mathbb{E}\left\{\left|\mathbf{a}_{m}^{H} \mathbf{D} \mathbf{a}_{m}^{*}\right|^{2}\left\|\mathbf{h}_{m}\right\|^{2}\right\}$, and $\mathbb{E}\left\{\left|\mathbf{a}_{m}^{H} \mathbf{D} \mathbf{a}_{m}^{*}\right|^{2}\left\|\mathbf{g}_{m}\right\|^{2}\right\}$. We have

$$
\begin{aligned}
& \mathbb{E}\left\{\left|\mathbf{a}_{m}^{H} \mathbf{D} \mathbf{a}_{m}^{*}\right|^{2}\left\|\mathbf{h}_{m}\right\|^{2}\right\}=4 \sum_{k=1}^{K} \mathbb{E}\left\{\left|\sum_{i=1}^{K} g_{m, i}^{*} h_{m, i}^{*} h_{m, k}\right|^{2}\right\} \\
& \stackrel{(a)}{=} 4 \sum_{k=1}^{K} \sum_{i=1}^{K} \mathbb{E}\left\{\left|g_{m, i}^{*} h_{m, i}^{*} h_{m, k}\right|^{2}\right\} \\
& \stackrel{(b)}{=} 8 \sum_{k=1}^{K} \alpha_{m, k}^{2} \beta_{m, k}+4 \sum_{k=1}^{K} \sum_{i \neq k}^{K} \alpha_{m, k} \alpha_{m, i} \beta_{m, i}
\end{aligned}
$$

where $(a)$ comes from the fact that $g_{m, i}^{*} h_{m, i}^{*} h_{m, k}, i=$ $1, \ldots, K$, are zero-mean mutual uncorrelated RVs, and (b) follows by using the identity $\mathbb{E}\left\{|x|^{2}\right\}=\sigma^{2}$ and $\mathbb{E}\left\{|x|^{4}\right\}=2 \sigma^{4}$, where $x \sim \mathcal{C N}\left(0, \sigma^{2}\right)$. Similarly, we obtain $\mathbb{E}\left\{\left|\mathbf{a}_{m}^{H} \mathbf{D} \mathbf{a}_{m}^{*}\right|^{2}\left\|\mathbf{g}_{m}\right\|^{2}\right\}=4 \sum_{k=1}^{K} \beta_{m, k}\left(\alpha_{m, k} \beta_{m, k}+c_{m}\right)$, and $\mathbb{E}\left\{\left|\mathbf{a}_{m}^{H} \mathbf{D} \mathbf{a}_{m}^{*}\right|^{2}\right\}=4 \sum_{k=1}^{K} \alpha_{m, k} \beta_{m, k}$. Thus, we get (4).

\section{B. Proof of Proposition 1}

From (11), we need to compute $\operatorname{Var}\left\{\sum_{m=1}^{M} p_{m, k}\right\}, \mathrm{MT}_{k}$, and $\mathrm{AN}_{k}$. Since $p_{m, k}, m=1, \ldots, M$, are independent, we have

$$
\operatorname{Var}\left\{\sum_{m=1}^{M} p_{m, k}\right\}=\sum_{m=1}^{M}\left(\mathbb{E}\left\{\left|p_{m, k}\right|^{2}\right\}-4 \gamma_{m}^{2} \alpha_{m, k}^{2} \beta_{m, k}^{2}\right) \text {. }
$$

By using the same technique as in Appendix A, we obtain

$$
\operatorname{Var}\left\{\sum_{m=1}^{M} p_{m, k}\right\}=4 \sum_{m=1}^{M} \gamma_{m}^{2} \alpha_{m, k} \beta_{m, k}\left(2 \alpha_{m, k} \beta_{m, k}+c_{m}\right) \text {. }
$$

Similarly, we obtain

$$
\begin{aligned}
\mathrm{MT}_{k} & =4 p_{\mathrm{S}} \sum_{j \neq k}^{K} \sum_{m=1}^{M} \gamma_{m}^{2} \alpha_{m, j} \beta_{m, k}\left(\alpha_{m, k} \beta_{m, k}+\alpha_{m, j} \beta_{m, j}+c_{m}\right) \\
& +4 p_{\mathrm{S}} \sum_{j=1}^{K} \sum_{m=1}^{M} \gamma_{m}^{2} \beta_{m, j} \beta_{m, k}\left(\alpha_{m, k} \beta_{m, k}+\alpha_{m, j} \beta_{m, j}+c_{m}\right) \\
& +4 p_{\mathrm{S}} \sum_{m=1}^{M} \gamma_{m}^{2} \beta_{m, k}^{2}\left(2 \alpha_{m, k} \beta_{m, k}+c_{m}\right) \\
\mathrm{AN}_{k} & =4 \sum_{m=1}^{M} \gamma_{m}^{2} \beta_{m, k}\left(\alpha_{m, k} \beta_{m, k}+c_{m}\right)+1
\end{aligned}
$$

Substituting (7), (20), (21), and (22) into (11), we obtain (14).

\section{REFERENCES}

[1] S. Fazeli-Dehkordy, S. Shahbazpanahi, and S. Gazor, "Multiple peer-topeer communications using a network of relays," IEEE Trans. Signal Process., vol. 57, no. 8, pp. 3053-3062, Aug. 2009.

[2] M. Fadel, A. El-Keyi, and A. Sultan, "QOS-constrained multiuser peerto-peer amplify-and-forward relay beamforming," IEEE Trans. Signal Process., vol. 60, no. 3, pp. 1397-1408, Mar. 2012.

[3] A. F. Dana and B. Hassibi, "On the power efficiency of sensory and ad-hoc wireless networks" IEEE Trans. Inf. Theory, vol. 52, no. 7, pp. 2890-2914, July 2006.

[4] C. Y. Leow, Z. Ding, K. Leung, and D. Goeckel, "On the study of analogue network coding for multi-pair, bidirectional relay channels," IEEE Trans. Wireless Commun., vol. 10, no. 2, pp. 670-681, Feb. 2011.

[5] M. Tao and R.Wang, "Linear precoding for multi-pair two-way MIMO relay systems with max-min fairness," IEEE Trans. Signal Process., vol. 60, no. 10 , pp. 5361-5370, Oct. 2012.

[6] T. Wang, B. P. Ng, Y. Zhang, and M. H. Er, "Multiple peer-to-peer communications for two-way relay networks," in Proc. Intern. Conf. Inf., Commun. Signal Process., Dec. 2011.

[7] J. Zhang, F. Roemer, and M. Haardt, "Distributed beamforming for twoway relaying networks with individual power constraints," in Proc. FortySixth Asilomar Conf. Signals, Syst. Comput. (ACSSC), Nov. 2012.

[8] C. Wang, H. Chen, Q. Yin, A. Feng, and A. Molisch, "Multi-user twoway relay networks with distributed beamforming," IEEE Trans. Wireless Commun., vol. 10, no. 10, pp. 3460-3471, Oct. 2011.

[9] F. Rusek, D. Persson, B. K. Lau, E. G. Larsson, T. L. Marzetta, O. Edfors, and F. Tufvesson, "Scaling up MIMO: Opportunities and challenges with very large arrays," IEEE Signal Process. Mag., vol. 30, no. 1, pp. 40-60, Jan. 2013.

[10] H. Cramér, Random Variables and Probability Distributions. Cambridge, UK: Cambridge University Press, 1970.

[11] H. Bölcskei, R. Nabar, O. Oyman, and A. J. Paulraj, "Capacity scaling laws in MIMO relay networks," IEEE Trans. Wireless Commun., vol. 5, no. 6, pp. 1433-1444, June 2006.

[12] R. Vaze and R. W. Heath, Jr., "Capacity scaling for MIMO two-way relaying," in Proc. IEEE Intern. Symp. Inf. Theory (ISIT), June 2007. 